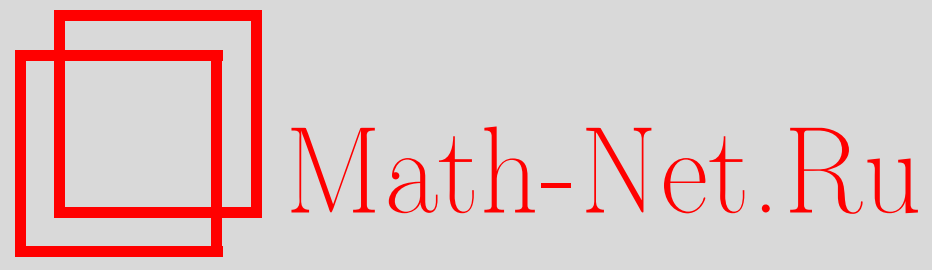

Ю. В. Немировский, Н. А. Фёдорова, Исследование рациональных структур криволинейного армирования в полярной системе координат, Вестн. Сам. гос. техн. ун-та. Сер. Физ.-мат. науки, 2013, выпуск 1(), 233244

DOI: https://doi.org/10.14498/vsgtu1164

Использование Общероссийского математического портала Math-Net.Ru подразумевает, что вы прочитали и согласны с пользовательским соглашением

http: //www .mathnet.ru/rus/agreement

Параметры загрузки:

IP: 54.197 .130 .99

26 апреля 2023 г., 14:03:07

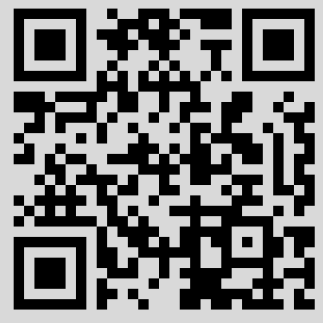


УДК 519.876:620.22-419.8

\title{
ИССЛЕДОВАНИЕ РАЦИОНАЛЬНЫХ СТРУКТУР КРИВОЛИНЕЙНОГО АРМИРОВАНИЯ В ПОЛЯРНОЙ СИСТЕМЕ КООРДИНАТ
}

\author{
Ю. В. Немировский ${ }^{1}$, Н. А. Фёдорова ${ }^{2}$ \\ 1 Институт теоретической и прикладной механики им. С. А. Христиановича СО РАН, \\ Россия, 630090, Новосибирск, ул. Институтская, 4/1. \\ 2 Институт космических и информационных технологий \\ Сибирского федерального университета, \\ Россия, 660074, Красноярск, ул. Киренского 26. \\ E-mails: nemirov@itam.nsc.ru, feodorova.natalia@mail.ru
}

На основе структурной модели решена задача рационального армирования криволинейными волокнами осесимметричной кольцевой пластины в полярной системе координат. Изучено влияние структурных параметров на предельное нагружение конструкиии.

Ключевые слова: структурная модель, криволинейное армирование.

1. Постановка задачи. Напряжённо-деформированное состояние армированной пластины в полярной системе координат $(\rho, \theta)$ относительно компонент тензоров деформаций $\varepsilon_{\rho}, \varepsilon_{\theta}, \varepsilon_{\rho \theta}$ и напряжений $\sigma_{\rho}, \sigma_{\theta}, \sigma_{\rho \theta}$ в осесимметрическом случае (искомые функции не зависят от полярного угла $\theta$ ) описывается приводимыми ниже соотношениями.

Уравнения равновесия имеют вид

$$
\frac{\partial \sigma_{\rho}}{\partial \rho}+\frac{\sigma_{\rho}-\sigma_{\theta}}{\rho}=0, \quad \frac{\partial \sigma_{\rho \theta}}{\partial \rho}+\frac{2}{\rho} \sigma_{\rho \theta}=0 .
$$

Пусть армирование выполнено $m$ семействами волокон $(m=1,2,3), \varphi_{m}-$ углы армирования, $\varepsilon_{m}$ - деформация в волокне, $\sigma_{m}$ - напряжение в волокне, $\omega_{m}$ - интенсивность армирования $m$-тым семейством волокон. Деформации в волокне в полярной системе определим по структурной модели [1]

$$
\varepsilon_{\rho} \cos ^{2} \varphi_{m}+\varepsilon_{\theta} \sin ^{2} \varphi_{m}+\varepsilon_{\rho \theta} \cos \varphi_{m} \sin \varphi_{m}=\varepsilon_{m}
$$

Соотношения Коши, связывающие компоненты тензора деформаций и компоненты вектора смещений $u_{\rho}, u_{\theta}$, в условиях осесимметричной деформации имеют вид

$$
\varepsilon_{\rho}=\frac{\partial u_{\rho}}{\partial \rho}, \quad \varepsilon_{\theta}=\frac{u_{\rho}}{\rho}, \quad \varepsilon_{\rho \theta}=\left(\frac{\partial u_{\theta}}{\partial \rho}-\frac{u_{\theta}}{\rho}\right) .
$$

Пусть $m^{*}$ - некоторое фиксированное число семейств армирующих волокон. Закон Гука для неоднородного армированного материала с числом се-

Юрий Владимирович Немировский (д.ф.-м.н., проф.), главный научный сотрудник, лаб. физики быстропротекающих процессов.

Наталья Александровна Федорова (к.ф.-м.н., доц.), доцент, каф. прикладной математики и компьютерной безопасности. 
мейств армирующих волокон $m^{*}$ запишем в виде

$$
\begin{aligned}
& \sigma_{\rho}=\Omega \frac{E}{1-\nu^{2}}\left(\varepsilon_{\rho}+\nu \varepsilon_{\theta}\right)+\sum_{m=1}^{m^{*}} \sigma_{m} \omega_{m} \cos ^{2} \varphi_{m}, \\
& \sigma_{\theta}=\Omega \frac{E}{1-\nu^{2}}\left(\varepsilon_{\theta}+\nu \varepsilon_{\rho}\right)+\sum_{m=1}^{m^{*}} \sigma_{m} \omega_{m} \sin ^{2} \varphi_{m}, \\
& \sigma_{\rho \theta}=\Omega \frac{E}{1+\nu} \varepsilon_{\rho \theta}+\sum_{m=1}^{m^{*}} \sigma_{m} \omega_{m} \cos \varphi_{m} \sin \varphi_{m}, \quad \Omega=1-\sum_{m=1}^{m^{*}} \omega_{m},
\end{aligned}
$$

где $E, \nu$ - соответственно модуль Юнга и коэффициент Пуассона связующего материала.

При наложении дополнительных условий постоянства сечений волокон [2] интенсивность армирования $\omega_{m} m$-тым семейством волокон удовлетворяет следующим условиям в полярной системе координат [3]:

$$
\frac{\partial}{\partial \rho}\left(\rho \omega_{m} \cos \varphi_{m}\right)+\frac{\partial}{\partial \theta}\left(\omega_{m} \sin \varphi_{m}\right)=0 .
$$

В рассматриваемой задаче интенсивность $\omega_{m}$ найдём из (4) после задания уравнений конкретных траекторий армирования $\rho=\rho(\theta)$, введения углов армирования $\varphi_{m}$ и начальных условий выхода арматуры.

2. Разрешающая система уравнений. Сформулируем задачу об осесимметричной деформации армированной пластины в перемещениях $u_{\rho}, u_{\theta}$. Для этого соотношения (3) подставим в уравнения равновесия (1), предварительно напряжения $\sigma_{m}$ в волокнах найдём по формулам

$$
\sigma_{m}=E_{m}\left(\varepsilon_{\rho} \cos ^{2} \varphi_{m}+\varepsilon_{\theta} \sin ^{2} \varphi_{m}+\varepsilon_{\rho \theta} \cos \varphi_{m} \sin \varphi_{m}\right)
$$

где $E_{m}-$ модуль Юнга материала $m$-того семейства волокон.

Напряжения $\sigma_{\rho}, \sigma_{\theta}, \sigma_{\rho \theta}$ с учётом структурных характеристик примут вид

$$
\begin{aligned}
\sigma_{\rho}=m_{1}\left(\varepsilon_{\rho}+\nu \varepsilon_{\theta}\right)+\sum_{m=1}^{m^{*}} E_{m} \omega_{m}\left(\varepsilon_{\rho} \cos ^{2} \varphi_{m}\right. & +\varepsilon_{\theta} \sin ^{2} \varphi_{m}+ \\
& \left.+\varepsilon_{\rho \theta} \sin \varphi_{m} \cos \varphi_{m}\right) \cos ^{2} \varphi_{m} \\
\sigma_{\theta}=m_{1}\left(\varepsilon_{\rho}+\nu \varepsilon_{\theta}\right)+\sum_{m=1}^{m^{*}} E_{m} \omega_{m}\left(\varepsilon_{\rho} \cos ^{2} \varphi_{m}\right. & +\varepsilon_{\theta} \sin ^{2} \varphi_{m}+ \\
& \left.+\varepsilon_{\rho \theta} \sin \varphi_{m} \cos \varphi_{m}\right) \sin ^{2} \varphi_{m} \\
\sigma_{\rho \theta}=m_{2} \varepsilon_{\rho \theta}+ & \sum_{m=1}^{m^{*}} E_{m} \omega_{m}\left(\varepsilon_{\rho} \cos ^{2} \varphi_{m}\right. \\
& +\varepsilon_{\theta} \sin ^{2} \varphi_{m}+ \\
& \left.+\varepsilon_{\rho \theta} \sin \varphi_{m} \cos \varphi_{m}\right) \sin \varphi_{m} \cos \varphi_{m}
\end{aligned}
$$


Соотношения для напряжений $\sigma_{\rho}, \sigma_{\theta}, \sigma_{\rho \theta}$ запишем в виде

$$
\begin{gathered}
\sigma_{\rho}=a_{11} \varepsilon_{\rho}+a_{12} \varepsilon_{\theta}+a_{13} \varepsilon_{\rho \theta}, \quad \sigma_{\theta}=a_{12} \varepsilon_{\rho}+a_{22} \varepsilon_{\theta}+a_{23} \varepsilon_{\rho \theta}, \\
\sigma_{\rho \theta}=a_{13} \varepsilon_{\rho}+a_{23} \varepsilon_{\theta}+a_{33} \varepsilon_{\rho \theta} \quad\left(m_{1}=\Omega \frac{E}{1-\nu^{2}}, m_{2}=\Omega \frac{E}{1+\nu}\right),
\end{gathered}
$$

где введены коэффициенты

$$
\begin{gathered}
a_{11}=m_{1}+\sum_{m=1}^{m^{*}} E_{m} \omega_{m} \cos ^{4} \varphi_{m}, \quad a_{12}=\nu m_{1}+\sum_{m=1}^{m^{*}} E_{m} \omega_{m} \cos ^{2} \varphi_{m} \sin ^{2} \varphi_{m} \\
a_{13}=\sum_{m=1}^{m^{*}} E_{m} \omega_{m} \cos ^{3} \varphi_{m} \sin \varphi_{m}, \quad a_{22}=m_{1}+\sum_{m=1}^{m^{*}} E_{m} \omega_{m} \sin ^{4} \varphi_{m}, \\
a_{23}=\sum_{m=1}^{m^{*}} E_{m} \omega_{m} \cos \varphi_{m} \sin ^{3} \varphi_{m}, \quad a_{33}=m_{2}+\sum_{m=1}^{m^{*}} E_{m} \omega_{m} \cos ^{2} \varphi_{m} \sin ^{2} \varphi_{m} .
\end{gathered}
$$

После подстановки (5) в уравнения равновесия (1) с учётом (2) получим относительно компонент перемещений следующую систему дифференциальных уравнений:

$$
\begin{aligned}
& a_{11} \frac{d^{2} u_{\rho}}{d \rho^{2}}+a_{13} \frac{d^{2} u_{\theta}}{d \rho^{2}}+\left(\frac{d a_{11}}{d \rho}+\frac{a_{11}}{\rho}\right) \frac{d u_{\rho}}{d \rho}+\left(-\frac{a_{23}}{\rho}+\frac{d a_{13}}{d \rho}\right) \frac{d u_{\theta}}{d \rho}+ \\
&+\left(\frac{1}{\rho} \frac{d a_{12}}{d \rho}-\frac{a_{22}}{\rho^{2}}\right) u_{\rho}+\left(-\frac{1}{\rho} \frac{d a_{13}}{d \rho}+\frac{a_{23}}{\rho^{2}}\right) u_{\theta}=0 \\
& a_{13} \frac{d^{2} u_{\rho}}{d \rho^{2}}+\frac{a_{33}}{2} \frac{d^{2} u_{\theta}}{d \rho^{2}}+\left(\frac{d a_{13}}{d \rho}+\frac{a_{23}}{\rho}+\frac{2 a_{13}}{\rho}\right) \frac{d u_{\rho}}{d \rho}+ \\
&+\left(-\frac{a_{33}}{\rho}+\frac{d a_{33}}{d \rho}+\frac{2 a_{33}}{\rho}\right) \frac{d u_{\theta}}{d \rho}+\left(\frac{1}{\rho} \frac{d a_{23}}{d \rho}+\frac{a_{23}}{\rho^{2}}\right) u_{\rho}+ \\
&+\left(-\frac{1}{\rho} \frac{d a_{33}}{d \rho}-\frac{a_{33}}{\rho^{2}}\right) u_{\theta}=0 .
\end{aligned}
$$

K системе (6) присоединим четыре граничных условия на внешнем и внутреннем контурах кольцевой пластины. Пусть на внутреннем контуре при $\rho=\rho_{1}$ заданы перемещения:

$$
u_{\rho}=C_{1}^{*}, \quad u_{\theta}=C_{2}^{*}
$$

(при $C_{1}^{*}=0, C_{2}^{*}=0$ имеем жёстко закрепленный вал, при $C_{1}^{*}=0, C_{2}^{*} \neq 0-$ возможно скручивание вала).

На внешнем контуре $\rho=\rho_{2}$ заданы радиальное и касательное усилия $p_{n}$, $p_{\tau}$. С учётом соотношений (5) и (2) условия на внешнем контуре примут вид

$$
\begin{aligned}
& a_{11} \frac{d u_{\rho}}{d \rho}+a_{12} \frac{u_{\rho}}{\rho}+\left.a_{13}\left(\frac{d u_{\theta}}{d \rho}-\frac{u_{\theta}}{\rho}\right)\right|_{\rho=\rho_{2}}=p_{n}, \\
& a_{13} \frac{d u_{\rho}}{d \rho}+a_{23} \frac{u_{\rho}}{\rho}+\left.a_{33}\left(\frac{d u_{\theta}}{d \rho}-\frac{u_{\theta}}{\rho}\right)\right|_{\rho=\rho_{2}}=p_{\tau} .
\end{aligned}
$$

Возможны следующие комбинации в граничных условиях: на внутреннем контуре задано одно из усилий и одно из перемещений, на внешнем- 
оставшееся усилие и перемещение. Система (6) и граничные условия (7), (8) представляют собой обобщённую двухточечную краевую задачу для системы обыкновенных дифференциальных уравнений. В граничные условия (7), (8) для общего случая армирования входят как обе неизвестные функции $u_{\rho}, u_{\theta}$, так и их производные. Коэффициенты системы содержат полный набор структурных характеристик материала: число $m^{*}$ семейств армирующих волокон, механические характеристики материалов связующего и волокна, интенсивность $\omega_{m}$ и тригонометрические функции углов армирования $\varphi_{m}$.

3. Армирование по спиралям. Выполним в рамках поставленной задачи армирование кольцевой пластины по криволинейным траекториям, которые являются семействами логарифмических и алгебраических спиралей [4]. Для построения разрешающей системы необходимо определить коэффициенты $a_{i j}$ системы (6). В каждом конкретном случае заданных семейств спиралей вычислим интенсивность $\omega_{m}$ и углы армирования $\varphi_{m}$.

1. Армирование по семействам логарифмических спиралей. Пусть дано семейство логарифмических спиралей вида $\rho=C b^{\theta}, C$ - параметр семейства, $b$ - параметр спирали, при $b>1$ спираль развертывается вокруг полюса против хода часовой стрелки (рис. 1 ), если $b<1$, то спираль закручивается по часовой стрелке (рис. 2). Вычислим угол армирования

$$
\operatorname{tg} \varphi=\frac{\rho}{\rho^{\prime}}=\frac{1}{\ln b},
$$

то есть для логарифмической спирали угол армирования - некоторая константа. Определим интенсивность армирования $\omega_{1}(\rho)$ из уравнения (4) при заданных углах армирования, в нем производную по $\theta$ вычисляем по формуле

$$
\frac{\partial}{\partial \theta}=\frac{\partial \rho}{\partial \theta} \frac{\partial}{\partial \rho}
$$

с учётом уравнения траектории $\rho=C b^{\theta}$. В результате имеем

$$
\frac{\partial}{\partial \rho}\left(\rho \omega_{1}\right)+\rho \frac{\partial}{\partial \rho}\left(\omega_{1}\right)=0 .
$$

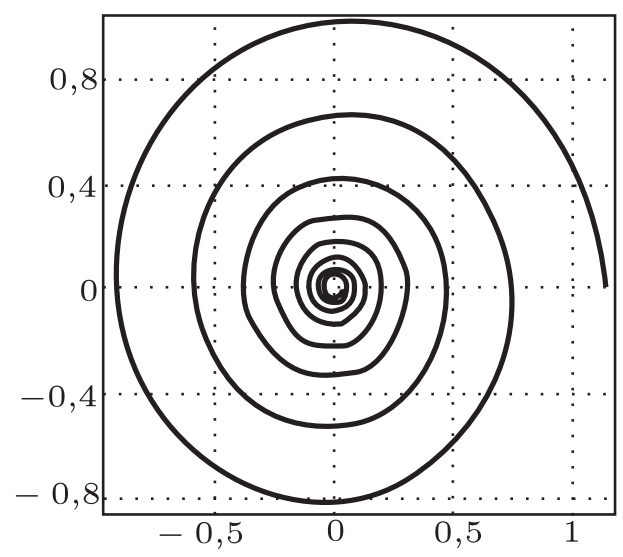

Рис. 1

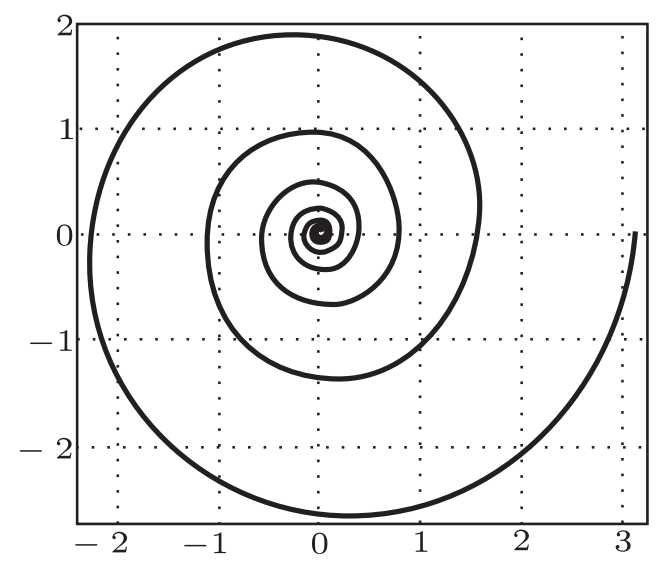

Рис. 2 
В (9) частную производную заменяем обычной производной по $\rho$, так как исключили зависимость от окружной координаты. Интегрируя (9) с учётом заданной интенсивности армирования $\omega_{0}$ на внутреннем контуре $\rho=\rho_{1}$, получим следующее выражение для интенсивности армирования по логарифмической спирали

$$
\omega_{1}=\omega_{0} \sqrt{\rho_{1} / \rho}
$$

2. Армирование по семействам спиралей Архимеда. Зададим спираль Архимеда $\rho=a \theta, a$ - коэффициент пропорциональности [4]. Иллюстрация кривой приведена на рис. 3. Угол армирования находим из соотношения $\operatorname{tg} \varphi=$ $=\theta=\rho / a$, вычисляем $\sin \varphi, \cos \varphi$ через $\operatorname{tg} \varphi$, после подстановки в (4) получаем следующее уравнение для интенсивности армирования $\omega_{1}(\rho)$ :

$$
\frac{\partial}{\partial \rho}\left(\rho \omega_{1} \frac{1}{\sqrt{1+\operatorname{tg} \varphi^{2}}}\right)=0
$$

оператор дифференцирования по окружной координате вдоль траектории армирования равен

$$
\frac{\partial}{\partial \theta}=a \frac{\partial}{\partial \rho} .
$$

Тогда интенсивность армирования в произвольной точке кольцевой пластины найдем по формуле

$$
\omega_{1}=C \sqrt{1+\operatorname{tg} \varphi} / \rho .
$$

Пусть на внутреннем контуре $\rho=\rho_{1}$ задан угол вхождения арматуры $\varphi_{0}$ и задана интенсивность армирования $\omega_{0}$, что соответствует условиям технологического процесса. После вычисления константы интегрирования из условий на внутреннем контуре пластины интенсивность армирования имеет вид

$$
\omega_{1}=\frac{\omega_{0} \sqrt{\rho_{1}^{2}+\rho^{2} \operatorname{tg}^{2} \varphi_{0}}}{\rho \sqrt{1+\operatorname{tg} \varphi^{2}}} .
$$

Для тангенса угла армирования получим выражение $\operatorname{tg} \varphi=\left(\rho / \rho_{1}\right) \operatorname{tg} \varphi_{0}$.

3. Спираль Ферма. Спираль Ферма задается уравнением $\rho^{2}=a^{2} \theta, a-$ коэффициент пропорциональности. Для тангенса угла армирования получим выражение $\operatorname{tg} \varphi=2 \rho^{2} / a^{2}$. С учётом условий на внутреннем контуре определяем параметр $a^{2}: a^{2}=2 \rho_{1} / \operatorname{tg} \varphi_{0}$, ограничение на условия на внутреннем контуре $\operatorname{tg} \varphi_{0}>0$.

Оператор дифференцирования по окружной координате вдоль траектории армирования определим по формуле

$$
\frac{\partial}{\partial \theta}=\frac{a^{2}}{2 \rho} \frac{\partial}{\partial \rho}
$$

Тогда уравнение для интенсивности армирования $\omega(\rho)$ в произвольной точке кольцевой пластины примет вид

$$
\frac{\partial}{\partial \rho} \frac{\rho \omega}{\sqrt{a^{4}+4 \rho^{4}}}+\frac{1}{\rho} \frac{\partial}{\partial \rho} \frac{\rho^{2} \omega}{\sqrt{a^{4}+4 \rho^{4}}}=0 .
$$


Найдём решение уравнения (10):

$$
\omega=C_{1} \frac{\sqrt{a^{2}+2 a \rho+2 \rho^{2}} \sqrt{a^{2}-2 a \rho+2 \rho^{2}}}{\sqrt{\rho^{3}}} .
$$

Сформулированные выше условия на внутреннем контуре дают соотношения для определения константы интегрирования, и окончательно для интенсивности армирования по спирали Ферма имеем

$$
\omega=\frac{\omega_{0} \sqrt{\rho_{1}^{3}}}{\sqrt{a^{2}+2 a \rho_{1}+2 \rho_{1}^{2}} \sqrt{a^{2}-2 a \rho_{1}+2 \rho_{1}^{2}}} \frac{\sqrt{a^{2}+2 a \rho+2 \rho^{2}} \sqrt{a^{2}-2 a \rho+2 \rho^{2}}}{\sqrt{\rho^{3}}},
$$

где $a^{2}=2 \rho_{1} / \operatorname{tg} \varphi_{0}$.

Вид траектории армирования по спирали Ферма приведен на рис. 4.

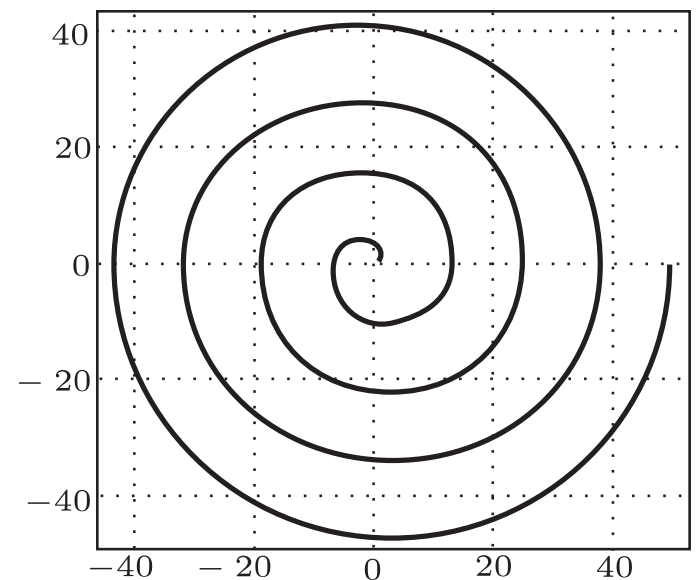

Рис. 3

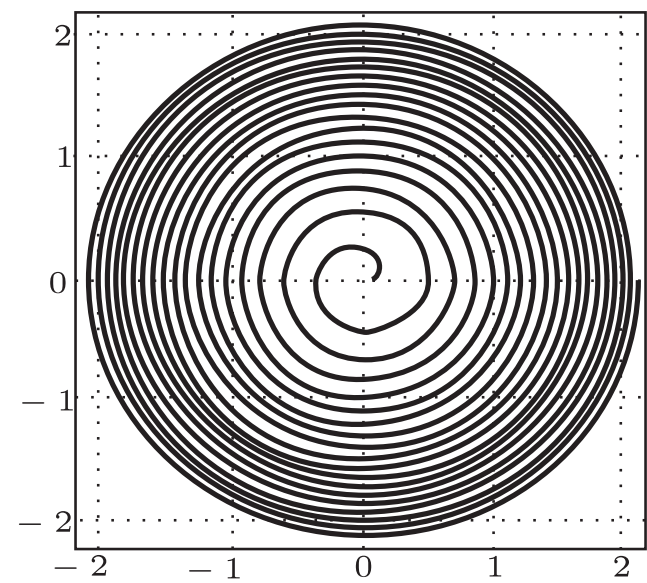

Рис. 4

4. «Велоколесо». Спицы велоколеса в полярной системе координат представляют семейство прямых, заданных уравнением $\rho=a / \sin \theta$, где $a-$ константа, параметр велоколеса, $-\pi<\theta<0, \theta \neq \pi / 2$. Вычислим $\rho_{\theta}^{\prime}$, найдём $\theta$ через $\rho$ из уравнения траектории: $\theta=\arcsin a / \rho$, получим выражение тангенса угла армирования $\operatorname{tg} \varphi=-\operatorname{tg} \theta$ через полярный радиус

$$
\operatorname{tg} \varphi=\mp \frac{a}{\sqrt{\rho^{2}-a^{2}}}
$$

Оператор дифференцирования по окружной координате вдоль траектории армирования равен

$$
\frac{\partial}{\partial \theta}=-\frac{a \cos \theta}{\sin ^{2} \theta} \frac{\partial}{\partial \rho}
$$

В результате условие постоянства сечений волокон примет вид

$$
\frac{\partial}{\partial \rho}\left(\omega_{1} \sqrt{\rho^{2}-a^{2}}\right)+\rho \sqrt{\rho^{2}-a^{2}} \frac{\partial}{\partial \rho}\left(\frac{\omega_{1}}{\rho}\right)=0 .
$$


Пусть $\rho_{1}$ - внутренний радиус кольцевой пластины, $\theta_{0}-$ заданный угол выхода, тогда $a=\rho_{1} \sin \theta_{0}$. С учетом условий на внутреннем контуре $\left.\omega_{1}\right|_{\rho=\rho_{1}}=\omega_{0}$ получим интенсивность армирования

$$
\omega_{1}=\frac{\omega_{0} \sqrt{\rho}\left(\rho_{1}^{2}-\left(\rho_{1} \sin \theta_{0}\right)^{2}\right)^{1 / 4}}{\sqrt{\rho_{1}}\left(\rho^{2}-\left(\rho_{1} \sin \theta_{0}\right)^{2}\right)^{1 / 4}} .
$$

Армирование вдоль спиц велоколеса для кольцевой пластины находит применение в современной промышленности. В настоящей работе рассматривается армирование по траекториям, представляющим комбинации спиц велоколеса и семейств рассмотренных выше спиралей.

5. Изогональное армирование. Наряду с криволинейными структурами армирования по спиралям, рассматриваемыми в кольцевых пластинах, строим изогональные траектории армирования (т.е. линии, пересекающие кривые данного однопараметрического семейства под одним и тем же заданным углом $\alpha=\operatorname{arctg} k)[5]$. Процедура нахождения изогональных траекторий к данным координатным линиям криволинейной ортогональной системы координат описана в монографии [6].

Для семейства логарифмических спиралей семейство изогональных к ним траекторий описывается уравнением вида

$$
\rho=C_{1} \exp \left(\frac{1-k}{1+k} \theta\right)
$$

где $C_{1}$ - произвольная константа, $k=\operatorname{tg} \alpha$. Иллюстрации армирования концентрического кольца по логарифмическим спиралям и изогональным им траекториям для значений $k=3, k=0,7$ приведены на рис. 5, 6, где параметр семейств траекторий принимает три значения, изогональные траектории изображены пунктирными линиями.

Для семейств спиралей Архимеда уравнение изогональных траекторий имеет вид

$$
\rho=C_{2} e^{-k \theta}(1+k \theta)^{2 / k},
$$

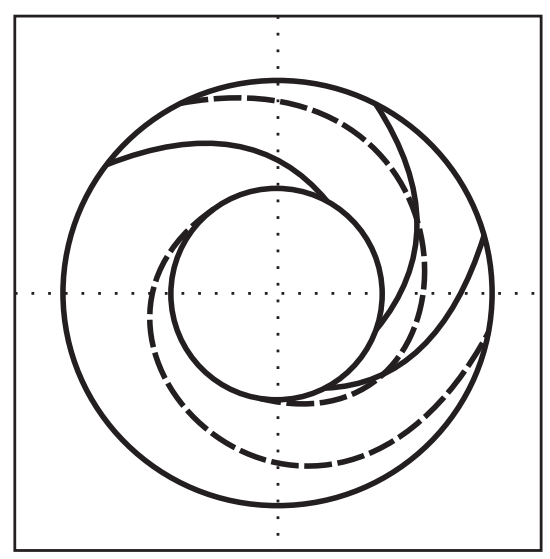

Рис. 5

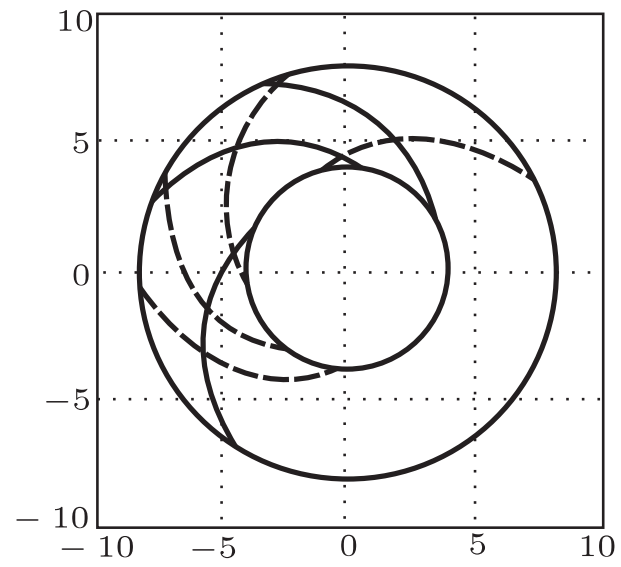

Рис. 6 


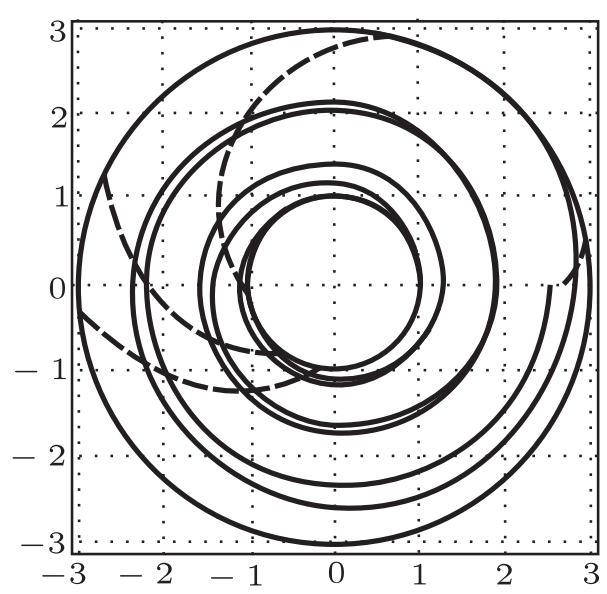

Рис. 7

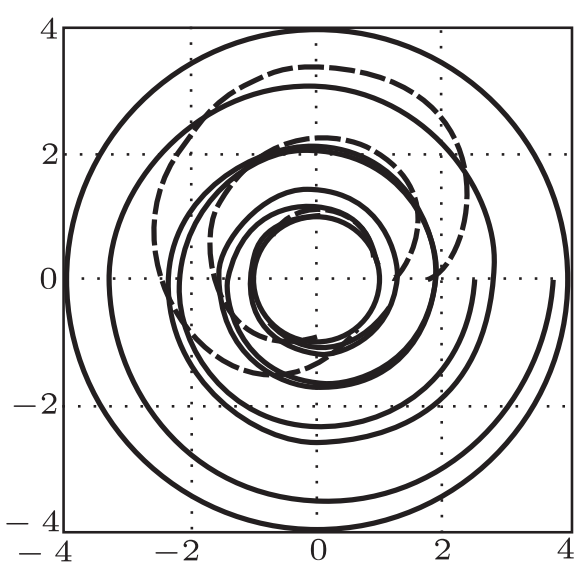

Рис. 8

где $C_{2}$ - произвольная константа. Армирование по спиралям Архимеда и изогональным им траекториям в кольцевой пластине для значений $k=0,7$, $k=1,4$ приведены на рис. 7,8 .

Коэффициенты системы (6) учитывают способы армирования семействами волокон в направлении любых изогональных траекторий, что дает широкое разнообразие структур армирования и позволяет в рамках единой схемы решения (6) получать композиционную конструкцию с заранее заданными свойствами.

4. Условия прочности армированного материала. Проверка условий разрушения упруго армированного материала имеет свои особенности [7]. Сформулируем кратко условия прочности армированного материала. Пусть материал изотропного связующего имеет различные пределы прочности при растяжении $\sigma_{c}^{+}$и сжатии $\sigma_{c}^{-}$. Тогда в случае плоского напряжённого состояния условие прочности Баландина для неоднородного материала через напряжения $\sigma_{\rho}^{c}, \sigma_{\theta}^{c}, \sigma_{\rho \theta}^{c}$ в связующем для полярной системы координат имеют вид

$$
\left(\sigma_{\rho}^{c}\right)^{2}+\left(\sigma_{\theta}^{c}\right)^{2}-\sigma_{\rho}^{c} \sigma_{\theta}^{c}+3\left(\sigma_{\rho \theta}^{c}\right)^{2}+\left(\sigma_{c}^{-}-\sigma_{c}^{+}\right)\left(\sigma_{\rho}^{c}+\sigma_{\theta}^{c}\right)<\sigma_{c}^{+} \sigma_{c}^{-}
$$

Левую часть соотношения (11) назовём функцией Баландина.

Для семейств армирующих волокон предполагаем, что пределы прочности (текучести) $m$-того семейства волокон при растяжении $\sigma_{m}^{+}$и сжатии $\sigma_{m}^{-}$ различны. Армирующие семейства волокон остаются упругими, если выполняются неравенства [8]

$$
-\sigma_{m}^{-}<E_{m}\left(\varepsilon_{\rho} l_{1 m}^{2}+2 \varepsilon_{\rho \theta} l_{1 m} l_{2 m}+\varepsilon_{\theta} l_{2 m}^{2}\right)<\sigma_{m}^{+}
$$

В (12) использованы обозначения $l_{m 1}=\cos \left(\varphi_{m}\right), l_{m 2}=\sin \left(\varphi_{m}\right)$, где $m-$ число семейств армирующих волокон, $E_{m}$ - модуль Юнга $m$-того семейства волокон. Таким образом, для проверки прочности армированного материала необходимо анализировать два условия: условие на прочность материала связующего (11) и условие на прочность армирующих волокон (12). 
На основании вышеизложенного следует ввести понятие предельного упругого состояния в некоторой точке рассматриваемой конструкции, по достижении которого хотя бы в одной точке либо в связующем, либо в волокне происходит выход за пределы упругости (напряжение превышает предел текучести). В данной точке может возникнуть микроразрушение.

5. Численное решение задачи. Численную схему строим относительно обезразмеренных переменных: линейный размер пластины относим к внешнему радиусу, в соотношениях для напряжений все величины относим к максимальному модулю Юнга армирующих семейств волокон. Система (6) и сформулированные граничные условия представляют собой обобщенную двухточечную краевую задачу для системы обыкновенных дифференциальных уравнений. Коэффициенты в (6) содержат полный набор структурных характеристик: число семейств армирующих волокон, механические характеристики материалов связующего и волокон, интенсивность и тригонометрические функции углов армирования.

Для численного решения обезразмеренная система (6) сводилась к системе четырёх дифференциальных уравнений первого порядка, затем строилась разностная схема, аппроксимирующая систему дифференциальных уравнений и краевые условия со вторым порядком точности. Полученная при этом система линейных уравнений с трехдиагональной матрицей коэффициентов решается методом ортогональной прогонки [9].

Постановка задачи свелась к реализации единой схемы, которая учитывает её разнообразные механические формулировки и различные способы армирования.

В численном эксперименте рассмотрена кольцевая пластина с тремя типами структур армирования: траекториями армирования являются семейства спиралей Архимеда и логарифмических спиралей (I) (рис. 9), семейство спиралей Архимеда и «спицы велоколеса» (II), семейство логарифмических спиралей и «спицы велоколеса» (III) (рис. 10). В качестве материала связующего выбран алюминий, армирование производится стальными волокнами. Механические характеристики металлокомпозита [10] и за-

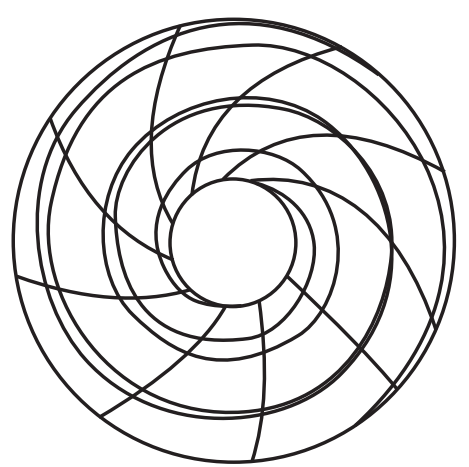

Рис. 9

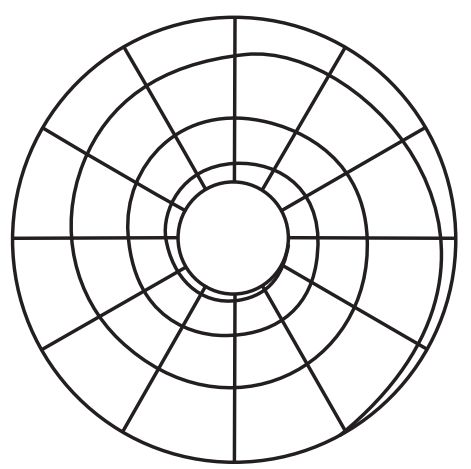

Рис. 10

Таблица 1

Механические и геометрические характеристики композита

\begin{tabular}{c|c|c|c|c|c|c|c}
\hline$A m p$ & $\nu$ & $E$, ГПА & $E_{1}$, ГПА & $E_{2}$, ГПА & $\omega_{01}$ & $\omega_{02}$ & $\operatorname{tg} \varphi_{0}$ \\
\hline$-2,0$ & 0,3 & 70,0 & 200,0 & 200,0 & 0,3 & 0,3 & 0,7 \\
$-1,5$ & 0,3 & 70,0 & 200,0 & 200,0 & 0,05 & 0,04 & 0,7 \\
0,5 & 0,3 & 70,0 & 200,0 & 200,0 & 0,32 & 0,1 & 0,7 \\
2,0 & 0,3 & 70,0 & 200,0 & 200,0 & 0,51 & 0,18 & 0,7 \\
\hline
\end{tabular}




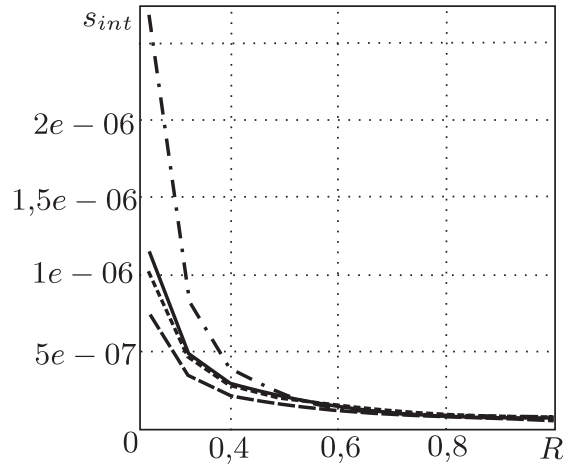

Рис. 11. Структура I

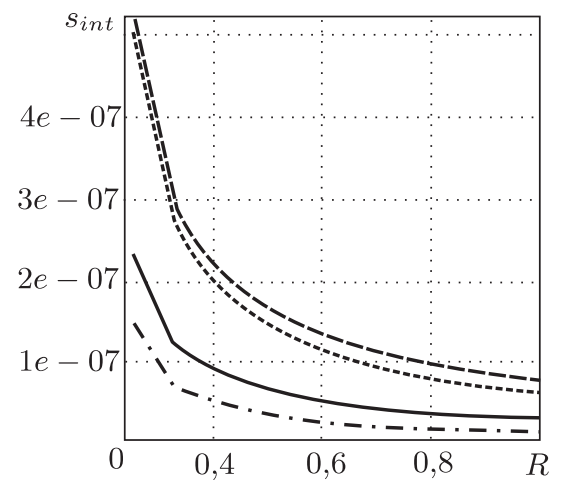

Рис. 13. Структура III

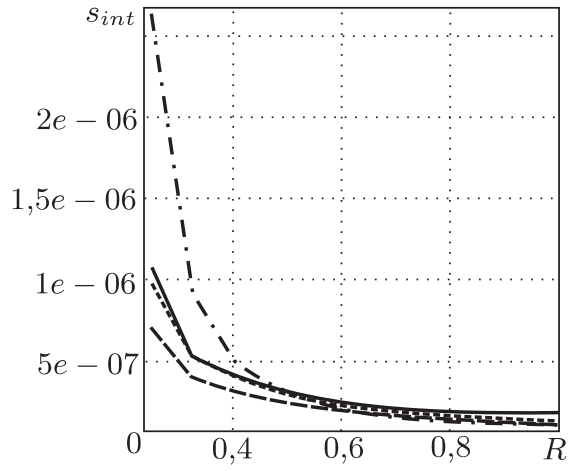

Рис. 12. Структура II

даваемые геометрические параметры конструкции представлены в табл. 1. Значения средней прочности для материала связующего $\sigma_{c}^{+}$составляет 0,29 ГПА, для стальных волокон пределы прочности при растяжении $\sigma_{1}^{+}=0,8$ ГПА, при сжатии $\sigma_{1}^{-}=0,4$ ГПА. На внутреннем контуре ставятся условия жесткой заделки. На внешнем контуре задаются нормальное и тангенциальное усилия $p_{n}, p_{\tau}$. В граничных условиях примем $p_{n} / p_{\tau}=A m p$, число $A m p$ назовём амплитудой внешней нагрузки, полагаем величину усилия $p_{n}=$ $=1$ МПА. Изучаем, как влияет изменение амплитуды на достижение предельной нагрузки согласно критериям (11), (12) для трёх рассматриваемых структур армирования.

На рис. 11-13 для четырёх типов начальных интенсивностей армирования $\omega_{01}, \omega_{02}$ при фиксированной внешней нагрузке показаны значения функции Баландина в безразмерных переменных. Как видим, структура III допускает значительное увеличение внешней нагрузки для всех начальных значений интенсивностей армирования, в отличие от двух других структур, в которых происходит приближение к предельному состоянию. Можем сделать вывод, что при фиксированном материале и уровне нагружения при заданных граничных условиях наиболее предпочтительной является структура армирования III.

Для анализа рассматриваемых структур армирования (I-III) введём характеристику, которую назовём степенью нагружения волокна $P$, определив её как отношение напряжений в волокне к пределу прочности соответствующего материала, выраженную в процентах. Задавшись значениями амплитуды внешней нагрузки и начальными условиями выхода арматуры, получим следующие зависимости $P$ для названных структур армирования (табл. 2).

Численные эксперименты показали, что за счёт управления геометрическими параметрами пластины и криволинейной укладкой семейств армирующих волокон можно получить конструкцию с заранее заданными свойствами. 


\section{Таблица 2}

\section{Степень нагружения волокна $P$, выраженная в процентах}

\begin{tabular}{c|c|c|c}
\hline структура & $\begin{array}{c}\omega_{01}=0,3, \\
\omega_{02}=0,3\end{array}$ & $\begin{array}{c}\omega_{01}=0,05, \\
\omega_{02}=0,0376\end{array}$ & $\begin{array}{c}\omega_{01}=0,1, \\
\omega_{02}=0,318\end{array}$ \\
\hline I & 6 & 40 & 6 \\
II & 9 & 70 & 10 \\
III & 14 & 80 & 22 \\
\hline
\end{tabular}

Предложенная методика позволяет прогнозировать поведение композита при различных вариантах механических свойств связующего и волокна, начальных стадий технологического процесса, выбора различных криволинейных траекторий армирования и их количества, размера внутреннего отверстия кольцевой пластины.

\section{БИБЛИОГРАФИЧЕСКИЙ СПИСОК}

1. Yu. V. Nemirovsky, "On the elastic-plastic behaviour of a reinforced layer" // Int. J. Mech. Sci., 1970. Vol. 12, no. 10. Pp. 898-903.

2. С. Б. Бушманов, Ю. В. Немировский, "Проектирование пластин, армированных равнонапряженными волокнами постоянного поперечного сечения" // Мех. композ. матер., 1983. № 2. C. 278-284; англ. пер.: S. B. Bushmanov, Yu. V. Nemirovskii, "Design of plates reinforced with equally stressed fibers of constant cross section" // Mech. Compos. Mater., 1983. Vol. 19, no. 2. Pp. 207-213.

3. Ю. В. Немировский, Н. А. Фёдорова, "Армирование плоских конструкций по криволинейным ортогональным траекториям"// Вестн. Сам. гос. техн. ун-та. Сер. Физ.-мат. науки, 2010. №5(21). C. 96-104. [Yu. V. Nemirovsky, N. A. Feodorova, "Reinforcement of Planar Structures along Orthogonal Curvilinear Trajectories" // Vestn. Samar. Gos. Tekhn. Univ. Ser. Fiz.-Mat. Nauki, 2010. no. 5(21). Pp. 96-104].

4. А. А. Гусак, Г. М. Гусак, Линии и поверхности. Минск: Выш. школа, 1985. 220 с. [A. A. Gusak, G. M. Gusak, Lines and surfaces. Minsk: Vysh. shkola, 1985. 220 pp.]

5. Н. А. Федорова, "Моделирование изогонально армированных кольцевых пластин в полярной системе координат" // Журн. СФУ. Сер. Матем. и физ., 2011. Т. 4, № 3. С. 400405. [N. A. Feodorova, "Modeling for reinforced with isogonal trajectories ring-shaped lamels in polar coordinate system" // J. Sib. Fed. Univ. Math. Phys., 2011. Vol.4, no. 3. Pp. 400405].

6. Ю. В. Немировский, Н. А. Фёдорова, Математическое моделирование плоских конструкций из армированных волокнистых материалов. Красноярск: СФУ, 2010. 136 с. [Yu. V. Nemirovskiy, N. A. Feodorova, Mathematical modeling of flat structures made of reinforced fiber materials. Krasnoyarsk: Sib. Fed. Univ., 2010. 136 pp.]

7. Ю. В. Немировский, Б. С. Резников, Прочность элементов конструкций из композитных материалов. Новосибирск: Наука, 1986. 165 с. [Yu. V. Nemirovskii, B. S. Reznikov, Strength of Structural Elements Made of Composite Materials. Novosibirsk: Nauka, 1986. $165 \mathrm{pp}$.

8. Ю. В. Немировский, "Об упруго-пластическом поведении армированного слоя" // ПМТФ, 1969. Т. 10, №6. C. 81-89. [Yu. V. Nemirovskii, "Elastoplastic behavior of a reinforced layer" // J. Appl. Mech. Tech. Phys., 1969. Vol. 10, no.6. Pp. 914-921].

9. J. M. Ortega, V. G. Pool, An introduction to Numerical Methods of Solving Differential Equations. New York: Pitman Publishing Inc., 1981; русск. пер.: Дж. Ортега, У. Пул, Введение в численные методы решения дифференциальных уравнений. М.: Наука, 1986. 288 c.

10. В. В. Васильев, В. Д. Протасов, В. В. Болотин и др., Композиционные материалы: Справочник. Москва: Машиностроение, 1990. 510 с. [V. V. Vasiliev, V. D. Protasov, 
V. V. Bolotin, et al., Composite Materials: A Handbook. Moscow: Mashinostroenie, 1990. 510 pp.]

Поступила в редакцию 14/XI/2012;

в окончательном варианте - 04/II/2013.

MSC: 74K99, 74E30

\title{
STUDY OF CURVILINEAR REINFORCEMENT RATIONAL STRUCTURES IN POLAR COORDINATE SYSTEM
}

\author{
Yu. V. Nemirovsky ${ }^{1}$, N.A. Feodorova ${ }^{2}$
}

1 Khristianovich Institute of Theoretical and Applied Mechanics,

Siberian Branch of the Russian Academy of Sciences,

4/1, Institutskaya st., Novosibirsk, 630090, Russia.

2 Institute of Space and Information Technologies,

Siberian Federal University,

26, Kirensky st., Krasnoyarsk, 660074, Russia.

E-mails: nemirov@itam.nsc.ru, feodorova.natalia@mail.ru

The problem of curvilinear fibers rational reinforcement for axially symmetric ringshaped lamel in polar coordinate system is solved by reference to the structural model. The effect of structural parameters for a construction limit stressing is studied.

Key words: structural model, curvelinear reinforcement.

Original article submitted 14/XI/2012; revision submitted 04/II/2013.

Yuriy V. Nemirovsky (Dr. Sci. (Phys. \& Math.)), Chief Scientist, Lab. of Fast Processes Physics. Nataliya A. Feodorova (Ph. D. (Phys. \& Math.)), Associate Professor, Dept. of Applied Mathematics \& Computer Security. 